Check for updates

Cite this: RSC Adv., 2018, 8, 24953

\section{Insights into the interaction mechanism between tiagabine hydrochloride and two serum albumins $\dagger$}

\begin{abstract}
Weiling Zhuo, ${ }^{a}$ Xilin Peng ${ }^{b}$ and Xiang Lin (D) *a
Tiagabine hydrochloride (TGB) is a widely used anticonvulsive drug for the treatment of epilepsy. To better understand the interactions of TGB with plasma proteins, human serum albumin (HSA) and bovine serum albumin (BSA) were selected as model proteins. TGB slightly increased thermal stability of the proteins as confirmed by VP-capillary differential scanning calorimetric (DSC) measurements. Isothermal titration calorimeter (ITC) results showed that TGB could be combined with HSA and BSA moderately, which was also corroborated by fluorescence analysis. Besides, the thermodynamic parameters $(\Delta H>0, \Delta S>0)$ indicated that hydrophobic forces played a major role in the formulation of TGB-HSA and TGB-BSA complexes. Moreover, the main binding sites of TGB to HSA and BSA were also examined by classical fluorescent probe (dansylsarcosine and dansylamide) experiments, showing that TGB and dansylsarcosine competitively interacted with HSA and BSA at the same binding sites. Additionally, TGB had no obvious effect on the conformation change of HSA and BSA as indicated by spectroscopic analyses. This study provides useful information about the interaction mechanism of TGB and serum albumins, which could help to better utilize TGB in biomedical field.
\end{abstract}

Received 16th May 2018

Accepted 29th June 2018

DOI: $10.1039 / \mathrm{c} 8 \mathrm{ra04153a}$

rsc.li/rsc-advances and bovine serum albumin (BSA) as model proteins to study their interactions with TGB. The BSA and HSA display approximately $76 \%$ sequence homology, and with different numbers and types of amino acids. HSA and BSA contain 585 and 583 amino acid residues, respectively, while HSA has only one tryptophan (Trp-214) residue, and BSA has two tryptophan (Trp134, 213) residues. ${ }^{9,10}$

In this study, several advanced analysis methods were performed to investigate TGB-HSA and TGB-BSA interactions. As many physiological functions of proteins depend on their intrinsic structure, a VP-capillary DSC measurement was performed to determine thermal stability of HSA and BSA when they interacted with or without TGB. Later, ITC was used to determine TGB-serum albumin binding ability, and fluorescence spectroscopy was utilized as an alternative method to further analyze their interactions. Site marker competitive

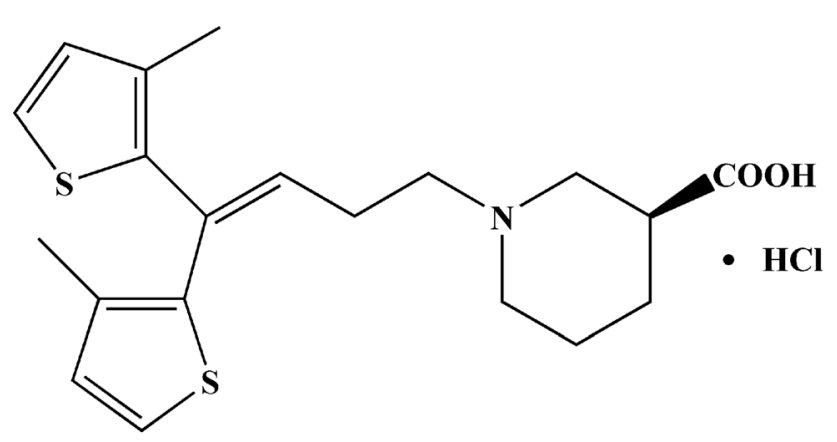

Fig. 1 Chemical structure of TGB.
${ }^{a}$ School of Chemical Engineering, Sichuan University, Chengdu 610065, People's Republic of China. E-mail: linxiang@scu.edu.cn; Fax: +86 028 85990908; Tel: +86 02885990908

${ }^{b}$ Sinopharm Chuankang Pharmaceutical Co., Ltd., Chengdu 611731, People's Republic of China

$\dagger$ Electronic supplementary information (ESI) available. See DOI: $10.1039 / \mathrm{c} 8 \mathrm{ra} 04153 \mathrm{a}$ for instance, binding mechanism, binding sites, etc. ${ }^{5,6}$ TGB ding with plasma proteins may affect its absorption, distriplays an important role in the transportation, deposition, and metabolism of numerous types of exogenous and endogenous compounds. ${ }^{8}$ This study chose human serum albumin (HSA) 
experiments were conducted to determine the potential main binding sites of TGB to HSA and BSA. Thereafter, the optimal binding models of TGB-HSA complex and TGB-BSA complex were simulated through molecular docking. Three-dimensional (3D) fluorescence spectroscopy and circular dichroism (CD) spectroscopy were adopted to monitor structural changes of the proteins affected by TGB. This study provides in-depth insights into the mechanism of the TGB-serum albumin binding interaction, which may be helpful for improving applications of TGB in clinical, and standardized screening in pharmaceutical firms and clinical research. ${ }^{\mathbf{1 1 2}}$

\section{Materials and methods}

\subsection{Chemicals and reagents}

HSA (fatty acid-free) was purchased from Sigma-Aldrich Company (St. Louis, USA). BSA (fatty acid-free, nuclease, and protease-free) was obtained from EMD Millipore Company (Billerica, USA). TGB (>98\% purity index) was acquired from Tokyo Chemical Industry Company (Tokyo, Japan). Dansylamide and dansylsarcosine were purchased from J\&K Scientific Ltd. (Beijing, China). Stock solutions of HSA, BSA, and TGB were prepared in phosphate buffer saline (PBS; 0.1 M, pH 7.40). The concentrations of HSA and BSA in buffer were corrected by the extinction coefficients of $35700 \quad \mathrm{M}^{-1} \quad \mathrm{~cm}^{-1}$ and $44720 \mathrm{M}^{-1} \mathrm{~cm}^{-1}$, respectively. ${ }^{13}$

\subsection{Differential scanning calorimetry measurements}

The VP-Capillary DSC measurement was run by means of a MicroCal VP-Capillary DSC (Malvern, Worcestershire, UK). Samples were prepared in PBS ( $\mathrm{pH}$ 7.4) and concentrations of HSA and BSA were maintained at $40 \mu \mathrm{M}$, whereas the molar ratios of TGB to HSA/BSA were $0: 1$ and $1: 1$. PBS was also used as a reference buffer. All the solutions were degassed properly under mild vacuum prior to the experiments. Scans were collected from $298 \mathrm{~K}$ to $363 \mathrm{~K}$ at a scan rate of $200 \mathrm{~K} \mathrm{~h}^{-1}$. Data were analyzed by using Origin 7.0 software provided by the instrument manufacturer to obtain the temperature at the midpoint of the unfolding transition $\left(T_{\mathrm{m}}\right)$.

\subsection{Isothermal titration calorimetry measurements}

ITC measurements were carried out on a Microcal ITC200 (Malvern, Worcestershire, UK) with an active cell volume of $185 \mu \mathrm{L}$. All titrations were performed in PBS at a stirring rate of $750 \mathrm{rpm}$ and using a $50 \mu \mathrm{L}$ titrating syringe at $298 \mathrm{~K}$. An automated sequence of 20 injections was used, with each of $2 \mu \mathrm{L}$ ligand (TGB) titration into the sample cell containing serum albumin. The intervals between each injection were spaced at $1.5 \mathrm{~min}$ for HSA samples and at 2 min for BSA samples to allow complete equilibration, which was performed with the equivalence point at the area midpoint of the titration. Titrations of TGB into buffer were performed as shown in Fig. S1. $\dagger$ The heat released or absorbed upon each injection was measured, and the data were plotted as integrated quantities. The resulting data were fitted to a one set of sites model and analyzed using MicroCal Origin 7.0 software, which was supplied by the instrument manufacturer.

\subsection{Fluorescence spectroscopy analyses}

Fluorescence analyses were conducted in a $1 \mathrm{~cm}$ quartz cell-using a Cary Eclipse fluorescence spectrophotometer (Varian, California, USA) at $298 \mathrm{~K}$. In a TGB-HSA system, the concentration of HSA was maintained at $2.4 \mu \mathrm{M}$, whereas the concentrations of TGB varied from $0 \mu \mathrm{M}$ to $28.8 \mu \mathrm{M}$ with a $4.8 \mu \mathrm{M}$ gradient growth based on preliminary experiments. In the TGB-BSA system, the concentration of BSA was $0.7 \mu \mathrm{M}$, and the concentration of TGB changed from $0 \mu \mathrm{M}$ to $16.8 \mu \mathrm{M}$ with a $2.4 \mu \mathrm{M}$ gradient growth. Fluorescence spectra were obtained using 5/10 nm (excitation/ emission) slit widths. The excitation wavelength was set at $280 \mathrm{~nm}$, and the emission range was from 300 to $450 \mathrm{~nm}$ unless specified otherwise. All emission intensities were corrected for inner filter effect, according to the following equation: ${ }^{\mathbf{1 4}}$

$$
F_{\text {corr }}=F_{\text {obs }} \times \mathrm{e}^{\frac{A_{\mathrm{ex}}+A_{\mathrm{em}}}{2}}
$$

where $F_{\text {corr }}$ and $F_{\text {obs }}$ are the corrected and observed fluorescence intensities, respectively. $A_{\mathrm{ex}}$ and $A_{\mathrm{em}}$ are the absorption values of the system at the excitation and emission wavelengths, respectively. Moreover, given that TGB consists of hydrochloric acid, to evaluate the effect of hydrochloric acid on HSA/BSA, 2.4 $\mu \mathrm{M}$ HSA containing $28.8 \mu \mathrm{M}$ hydrochloric acid was compared with free HSA and $0.7 \mu \mathrm{M}$ BSA containing $16.8 \mu \mathrm{M}$ hydrochloric acid was compared with free BSA using fluorescence, respectively. No obvious changes were observed in the spectral profiles obtained (Fig. S2†).

$3 \mathrm{D}$ fluorescence spectroscopy was performed at an excitation wavelength range from 200 to $400 \mathrm{~nm}$ in increments of $5 \mathrm{~nm}$. Emission spectra were monitored between 200 and $450 \mathrm{~nm}$. Concentrations of HSA and BSA were maintained at $2.4 \mu \mathrm{M}$ and $0.7 \mu \mathrm{M}$, respectively. The molar ratios of TGB to HSA/BSA were $0: 1$ and $6: 1$, respectively.

Site marker competitive experiments were conducted using dansylsarcosine and dansylamide as probes at $298 \mathrm{~K}$. All solutions were investigated at an exitation wavelength of $350 \mathrm{~nm}$. The emission spectra were recorded in the range of 380-670 $\mathrm{nm}$.

Fluorescence lifetime was measured on a Jobin Yvon Fluorolog-3 spectrofluorometer (Horiba, LesUlis, FRA). Timeresolved HSA/BSA fluorescence quenching by TGB was recorded by maintaining the excitation wavelength and emission wavelength at 280 and $345 \mathrm{~nm}$, respectively. The concentration of HSA was fixed at $2.4 \mu \mathrm{M}$, and the concentration of BSA was 0.7 $\mu \mathrm{M}$. The molar ratios of TGB to HSA/BSA were $0: 1$, and $12: 1$, respectively. Data were analyzed through the tail fitting method. The fit qualities were assessed based on $\chi^{2}$ values and residuals. The amplitude-weighted lifetime $(\langle\tau\rangle)$ for biexponential iterative fitting was calculated based on decay times and pre-exponential factors $(\alpha)$ in accordance with the following equation: ${ }^{15}$

$$
\langle\tau\rangle=\tau_{1} \alpha_{1}+\tau_{2} \alpha_{2}+\tau_{3} \alpha_{3}
$$

\subsection{Molecular docking analysis}

The molecular docking calculations were adopted with an AutoDock 4.2.5.1 program between ligand (TGB) and receptor 
(HSA/BSA). The 3D structure of TGB was acquired with Chemidraw 11.0 and optimized by using the Discover Minimization tool of Materials Studio 6.0 software (Accelrys, California, USA). Crystal structures of HSA and BSA were obtained from the Protein Data Bank (PDB), selecting the PDB IDs 2BXG for HSA and 4OR0 for BSA for docking simulation. ${ }^{16,17}$ Both crystal structures were optimized by adding polar hydrogen atoms, removing water molecules and ions, and adding and assigning Kollman united-atom partial charges. Docking was conducted by setting the grid box size at $126 \AA \times 126 \AA \times 126 \AA$ along with the $x, y$, and $z$ axes and with $0.619 \AA$ grid spacing. The center of the grid was set to 24.165, 8.326, and 17.384. MGLTools 1.5.6 AutoDockTools with AutoGrid 4 and AutoDock 4 were performed to set up and conduct docking calculations of the ligand and receptor. Docking was performed using the Lamarckian genetic algorithm in AutoDock. Global optimization started with 200 runs in 2500000 energy evaluations, with a maximum of 27000 generations. Binding models with the lowest binding free energy and most cluster members were used for further analyses.

\subsection{Circular dichroism spectroscopy}

CD spectra were recorded on a Chirascan-plus circular dichroism spectrometer (Applied Photophysics, Surrey, UK) at $298 \mathrm{~K}$. Concentrations of HSA and BSA were $2 \mu \mathrm{M}$, and that of TGB was $24 \mu \mathrm{M}$. Spectra were obtained in a $1 \mathrm{~mm}$ quartz cell in a range of 195 to $250 \mathrm{~nm}$ with a $1 \mathrm{~nm}$ step size, $1 \mathrm{~nm}$ band width, and $0.5 \mathrm{~s}$ point $^{-1}$ scanning speed and each spectrum was the average of three scans.

\section{Results and discussion}

\subsection{Evaluation of the thermal stability changes of HSA and BSA}

VP-DSC is a highly sensitive and high-throughput technique for characterizing thermal stability of proteins and ligandproteins. ${ }^{18}$ For an equilibrium in the unfolding process of a protein, changes in its midpoint of the unfolding transition $\left(T_{\mathrm{m}}\right)$, as a result of ligand binding, can be used to estimate shifts in thermal stability of the protein. ${ }^{19}$ Fig. 2 depicts the thermograms of TGB-HSA and TGB-BSA at molar ratios of $0: 1$ and $1: 1$. In Fig. $2 \mathrm{a}$, the HSA $(40 \mu \mathrm{M})$ is unfolded and gives a single endothermic peak with a $T_{\mathrm{m}}$ of $337.1 \mathrm{~K}$. After addition of TGB $(40 \mu \mathrm{M})$, the $T_{\mathrm{m}}$ increases to $337.5 \mathrm{~K}$. In Fig. $2 \mathrm{~b}$, the BSA $(40 \mu \mathrm{M})$ solution containing TGB $(40 \mu \mathrm{M})$ exhibits a single endothermic peak with a $T_{\mathrm{m}}$ of $335.8 \mathrm{~K}$, compared with $335.2 \mathrm{~K}$ in TGB-free solution. Thus, the $T_{\mathrm{m}}$ increment of HSA and BSA after TGB addition indicated that the thermal stability of proteins slightly increased after binding with TGB.

\subsection{Characterization of the TGB-HSA and TGB-BSA binding}

ITC is a direct and common technique for investigating biomolecular interactions, evaluating thermodynamics of ligand-protein interactions and studying the binding constant. ${ }^{20,21}$ Here, the ITC has been used to study the combination of TGB-HSA and TGB-BSA, respectively. The
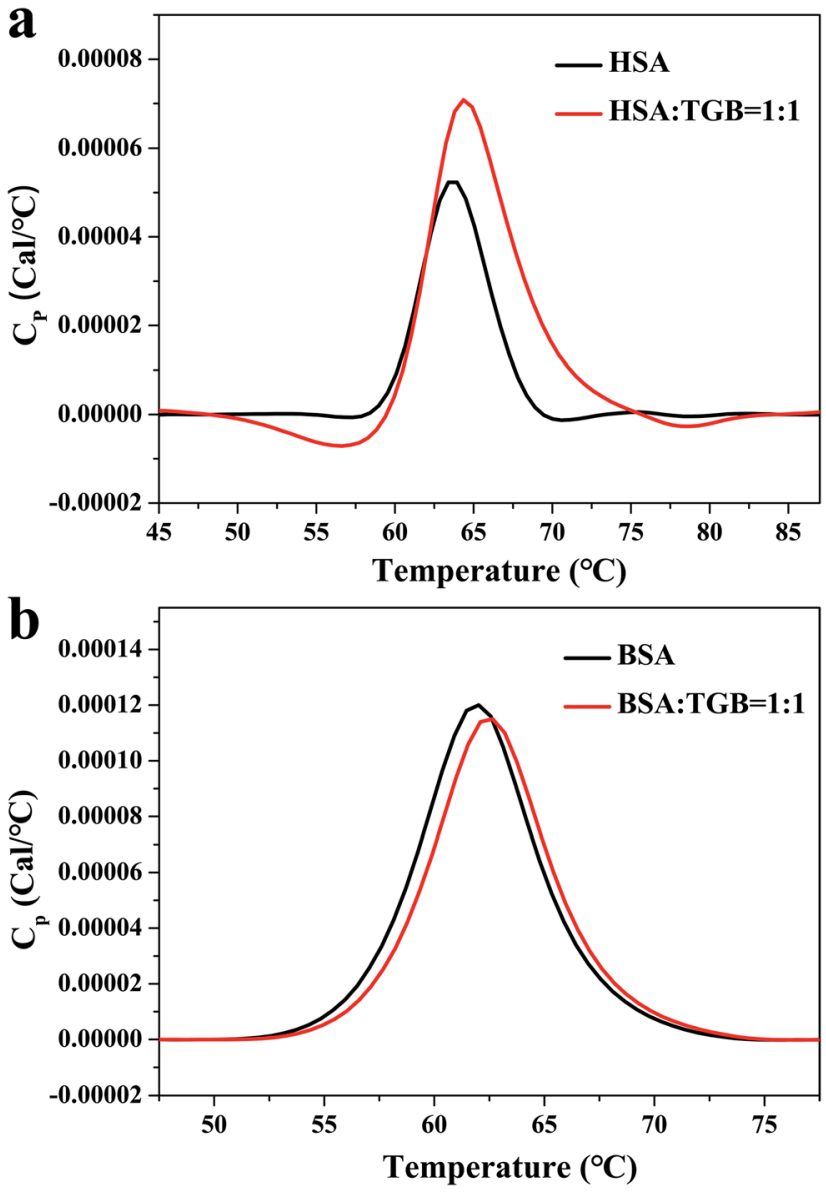

Fig. 2 DSC thermograms of TGB-HSA (a) and TGB-BSA (b) systems at $\mathrm{pH} 7.40$ and $298 \mathrm{~K}$, respectively. The concentrations of HSA and BSA are both $40 \mu \mathrm{M}$, and the molar ratios of TGB to HSA/BSA are $0: 1$ and $1: 1$.

calorimetric titration data at $\mathrm{pH} 7.4$ and $298 \mathrm{~K}$ are shown in Fig. 3. In Fig. 3, the upper panel shows the raw ITC results, where each of the heat burst peaks corresponded to a single injection of TGB into a HSA/BSA solution in the calorimeter cell. The lower panel shows an integrated heat profile that has been appropriately corrected by subtracting the corresponding dilution heats. ${ }^{22,23}$ In the lower panel, heat data are plotted against the corresponding molar ratio and fitted to a one set of sites model using MicroCal Origin 7.0 software, which was supplied by the instrument manufacturer. ${ }^{24}$ These data can be utilized to calculate the binding constant $(K)$, and the thermodynamics of binding, i.e., enthalpy changes $(\Delta H)$ and entropy changes $(\Delta S)$. The fitting curve is shown as a red curve in the lower panel of Fig. 3, whose amplitude is proportional to the change of enthalpy $(\Delta H)$, and where the slope of inflection point yields the binding constant $(K)$ in case of a sigmoidal titration curve. ${ }^{25}$ Obtained from ITC analyses, it revealed that the values of $K$ were $6.35 \pm 1.4 \times 10^{4} \mathrm{M}^{-1}$ for the TGB-HSA system and $3.67 \pm 2.89$ $\times 10^{4} \mathrm{M}^{-1}$ for TGB-BSA system, implying that binding abilities of the two systems were both moderate compared to that of other strong ligand-protein interactions with binding constants larger than $10^{6} \mathrm{M}^{-1} \cdot{ }^{26}$

The fluorescence spectroscopy method that is usually used for studying ligand-protein interactions, ${ }^{27-29}$ has also been 

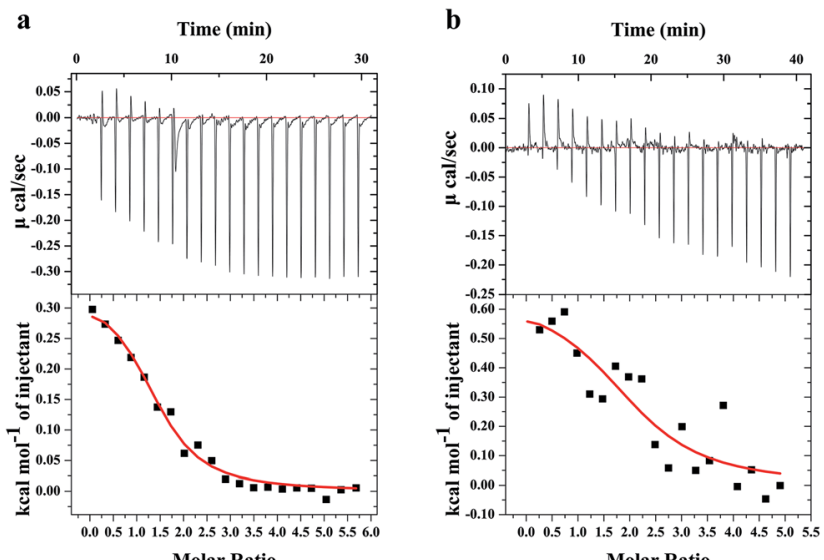

Fig. 3 ITC profiles for the titration of TGB with HSA (a) and BSA (b) at $\mathrm{pH} 7.40$ and $298 \mathrm{~K}$, respectively. The upper panel represents raw signals for the calorimetric titration of TGB with HSA/BSA, with a concentration of $2 \mathrm{mM}$ for TGB, $73.1 \mu \mathrm{M}$ for has, and $80.0 \mu \mathrm{M}$ for BSA. The lower panel shows integrated heat data after correcting for heat of TGB dilution. The solid line represents the best nonlinear least-squares fit to a one set of sites model.

applied to study binding ability between TGB and HSA/BSA. TGB can quench the intrinsic fluorescence of HSA and BSA to varying degrees, respectively (Fig. 4). This fluorescence quenching could reflect any process that decreases the fluorescence intensity of a fluorophore. Molecular interactions, including collisional quenching, ground-state complex formation, excited-state reactions, molecular rearrangements, and energy transfer, can induce fluorescence quenching. ${ }^{30}$ In this study, the fluorescence quenching mechanism of TGB with HSA and BSA were both static quenching, as the fluorescence lifetimes $\langle\tau\rangle$ of HSA and BSA were nearly unchanged in the presence of TGB (Fig. 4, Table 1).

Then, fluorescence quenching was analyzed according to the well-known Stern-Volmer equation:

$$
\frac{F_{0}}{F}=1+K_{\mathrm{Sv}}[Q]
$$

where $F_{0}$ and $F$ are the fluorescence intensities in the absence and presence of the quencher, respectively. For static quenching, $K_{\mathrm{SV}}$ is the association constant of the quencher-fluorophore ground-state complex..$^{31,32}$ Plots of $F_{0} / F$ against $1 /[\mathrm{Q}]$ of TGB quencher with HSA and BSA are shown in Fig. S3. $\uparrow$ The values of $K_{\mathrm{SV}}$ in the quenching process of serum albumin induced by TGB were calculated from slope and intercept of the curves. The calculated values of $K_{\mathrm{SV}}$ were $2.18 \pm 0.04 \times 10^{4} \mathrm{M}^{-1}$ for the TGB-HSA complex and $2.42 \pm 0.08 \times 10^{4} \mathrm{M}^{-1}$ for the TGB-BSA complex, respectively, indicating a moderate binding of the two complexes, which is inconsistent with the aforementioned ITC results.

In addition to the binding constant, thermodynamic parameters can also be obtained from ITC analysis. ${ }^{33}$ The positive values of $\Delta H\left(331.0 \pm 17.9 \mathrm{cal} \mathrm{mol}^{-1}\right)$ and $\Delta S(23.1 \pm 0.4$ cal $\mathrm{mol}^{-1} \mathrm{~K}^{-1}$ ) indicated that hydrophobic interactions played major roles in the formation of the TGB-HSA complex. ${ }^{34}$ Moreover, the Gibbs free energy change $(\Delta G)$ can be calculated by the van't Hoff equation $(\Delta G=\Delta H-T \Delta S)$. The negative $\Delta G$ $\left(-6.6 \pm 0.1 \mathrm{kcal} \mathrm{mol}^{-1}\right)$ implied that the binding process of TGB with HSA was spontaneous. In the TGB-BSA system, $\Delta H$ and $\Delta S$ were $647.0 \pm 139.7 \mathrm{cal} \mathrm{mol}^{-1}$ and $23.1 \pm 1.6 \mathrm{cal} \mathrm{mol}^{-1} \mathrm{~K}^{-1}$, respectively and the $\Delta G$ was calculated to be $-6.2 \pm$ $0.5 \mathrm{kcal} \mathrm{mol}^{-1}$. Thus, the binding force between TGB and HSA/ BSA was dominant with a hydrophobic interaction, and the binding process was spontaneous.

Crystallographic reports revealed that serum albumin, a heartshaped and highly helical structure protein, possesses multiple binding sites. Both HSA and BSA consist of a single polypeptide chain in three structurally homologous $\alpha$-helical domains (I-III), and each domain is divided into two subdomains (A and B). Two main binding sites named Sudlow site I and II, in subdomains IIA and IIIA, respectively, are highly adaptable for binding many widely accepted drugs. ${ }^{35}$ To investigate whether TGB could bind to HSA/BSA at the two primary binding sites or not, site marker competitive experiments were performed.
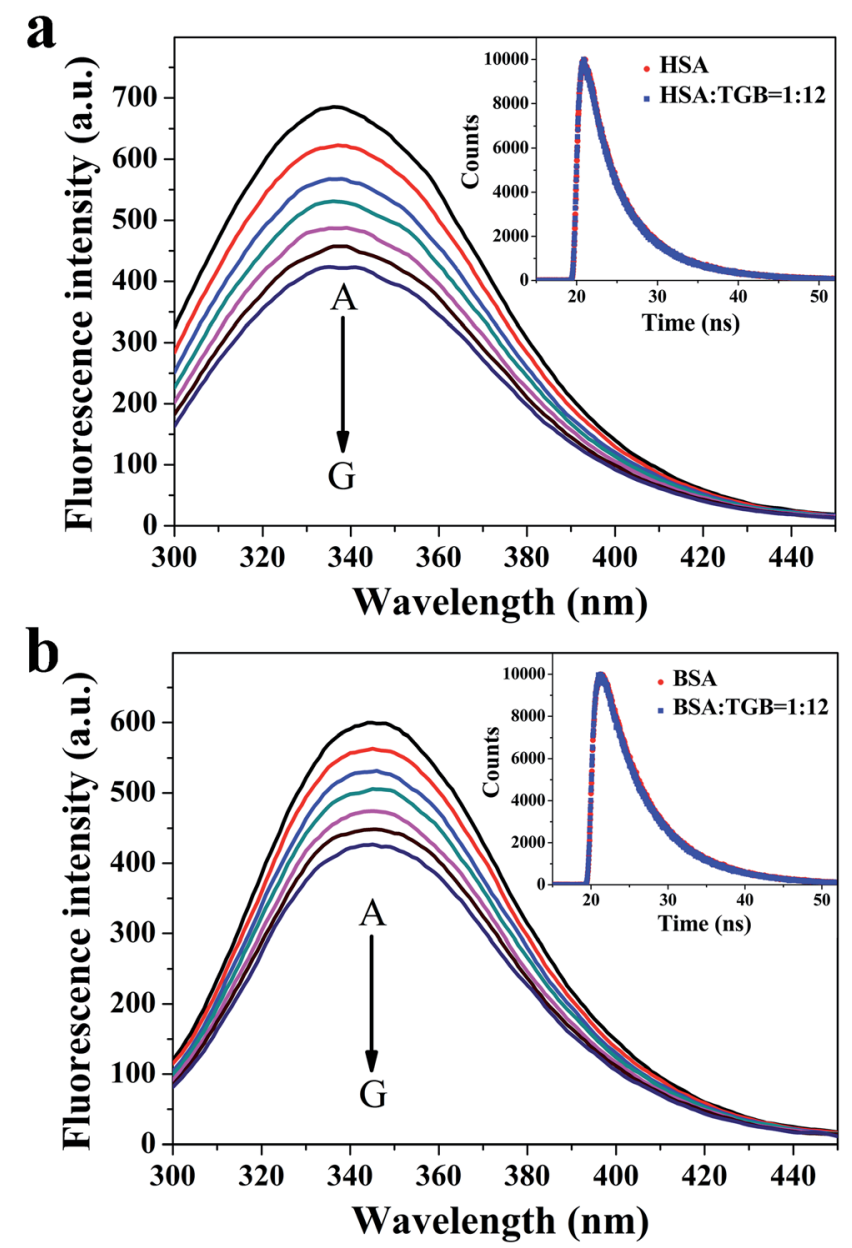

Fig. 4 Fluorescence spectra of HSA (a) and BSA (b) in the absence and presence of TGB at $\mathrm{pH} 7.40$ and $298 \mathrm{~K}$, respectively. The concentration of HSA is $2.4 \mu \mathrm{M}(\mathrm{A})$, and the concentrations of TGB are $4.8(B), 9.6(\mathrm{C})$, $14.4(D), 19.2(E), 24.0(F)$, and $28.8 \mu M(G)$. The concentration of BSA is $0.7 \mu \mathrm{M}(\mathrm{A})$, and the concentrations of TGB are $2.8(\mathrm{~B}), 5.6$ (C), 8.4 (D), $11.2(E), 14.0(F)$, and $16.8 \mu \mathrm{M}(\mathrm{G})$. The insets are the time-resolved fluorescence decay of HSA (a) and BSA (b) in the absence and presence of TGB. The concentrations of HSA and BSA are $2.4 \mu \mathrm{M}$ and $0.7 \mu \mathrm{M}$, respectively, and the molar ratios of TGB to HSA/BSA are $0: 1$ and $12: 1$ 
Table 1 Lifetime of HSA and BSA fluorescence decay with or without TGB

\begin{tabular}{|c|c|c|c|c|c|c|c|c|c|}
\hline System & Protein : drug & $\tau_{1}$ & $\tau_{2}$ & $\tau_{3}$ & $\alpha_{1}$ & $\alpha_{2}$ & $\alpha_{3}$ & $\langle\tau\rangle$ & $\chi^{2}$ \\
\hline \multirow[t]{2}{*}{ TGB-HSA } & $0: 1$ & 3.030 & 0.491 & 6.853 & 0.328 & 0.046 & 0.626 & 5.305 & 1.055 \\
\hline & $12: 1$ & 2.994 & 0.440 & 6.906 & 0.338 & 0.045 & 0.617 & 5.292 & 1.083 \\
\hline & $12: 1$ & 0.701 & 3.970 & 6.980 & 0.011 & 0.224 & 0.765 & 6.237 & 0.993 \\
\hline
\end{tabular}

\subsection{Identification of the main binding sites of TGB to HSA and BSA}

As early as the 1970 s, Sudlow et al. used fluorescent probes to identify the above two high-affinity binding sites, confirming that dansylsarcosine and ibuprofen were shown to bind specifically to Sudlow site II, while dansylamide, warfarin, and phenylbutazone were bound specifically to Sudlow site $\mathrm{I}^{36,37}$ At the excitation wavelength of $350 \mathrm{~nm}$, the HSA, BSA, or TGB cannot emit fluorescence, while dansylsarcosine or dansylamide only exclusively exhibit weak fluorescence, but the fluorescent intensity becomes stronger in the presence of HSA/BSA (Fig. 5). Here, dansylsarcosine and dansylamide were selected as specific fluorescent probes for the two distinct binding sites of TGB to HSA and BSA. Any combination of the single, binary, and ternary systems of the probe (dansylsarcosine/ dansylamide), the protein (HSA/BSA), and the drug (TGB) were performed using a fluorescence spectrometry method $\left(\lambda_{\mathrm{ex}}=350\right.$ $\mathrm{nm})$. In the present study, the molar ratios of protein : probe : TGB were $1: 4: 24$.
In Fig. 5a, the fluorescence intensity of dansylsarcosine-HSATGB is weaker than that of dansylsarcosine-HSA. We hypothesized that the original balance of the binary complex was interrupted by TGB, and a competitive binding with HSA might occur between dansylsarcosine and TGB, which causes liberation of some dansylsarcosine molecules from the dansylsarcosine-HSA complex in the presence of TGB, while TGB and HSA formed a ground-state complexation without fluorescence. Thus, the fluorescence intensity of dansylsarcosine-HSA was reduced after addition of TGB. These results indicated that TGB and dansylsarcosine could bind to the same binding site (Sudlow site II) in HSA, while the addition of TGB could partially replace dansylsarcosine that is bound to HSA. By contrast, as shown in Fig. 5b, the emission intensity of dansylamide-HSA-TGB is nearly the same as that of dansylamideHSA. This indicated that TGB, which exerted limited influence on the dansylamide-HSA complex, could not compete with dansylamide. Given the above, the TGB could bind to Sudlow site II (subdomain IIIA) of HSA.

Similarly, in the site maker competitive experiment of dansylsarcosine/dansylamide-BSA-TGB (Fig. 5c and d), the addition
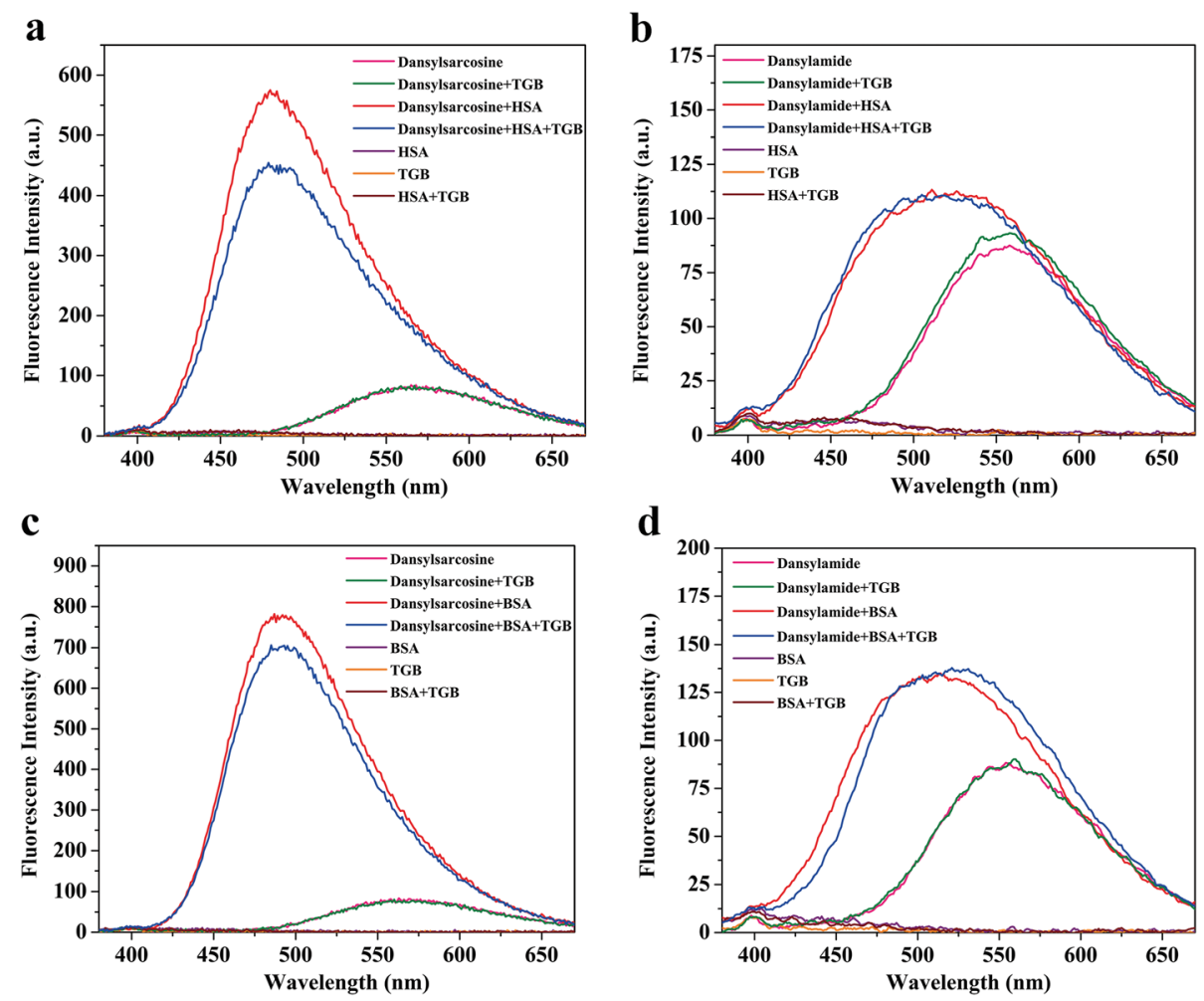

Fig. 5 At the excitation wavelength of $350 \mathrm{~nm}, \mathrm{pH} 7.40$, and $298 \mathrm{~K}$, the fluorescence emission spectra for (a) dansylsarcosine-HSA-TGB, (b) dansylamide-HSA-TGB, (c) dansylsarcosine-BSA-TGB, and (d) dansylamide-BSA-TGB. $C_{(\mathrm{HSA})}=C_{(\mathrm{BSA})}=2.4 \mu \mathrm{M}, C_{(\text {Dansylsarcosine })}=C_{(\text {Dansylamide })}=$ $9.6 \mu \mathrm{M}, \mathrm{C}_{(\mathrm{TGB})}=57.6 \mu \mathrm{M}$. 

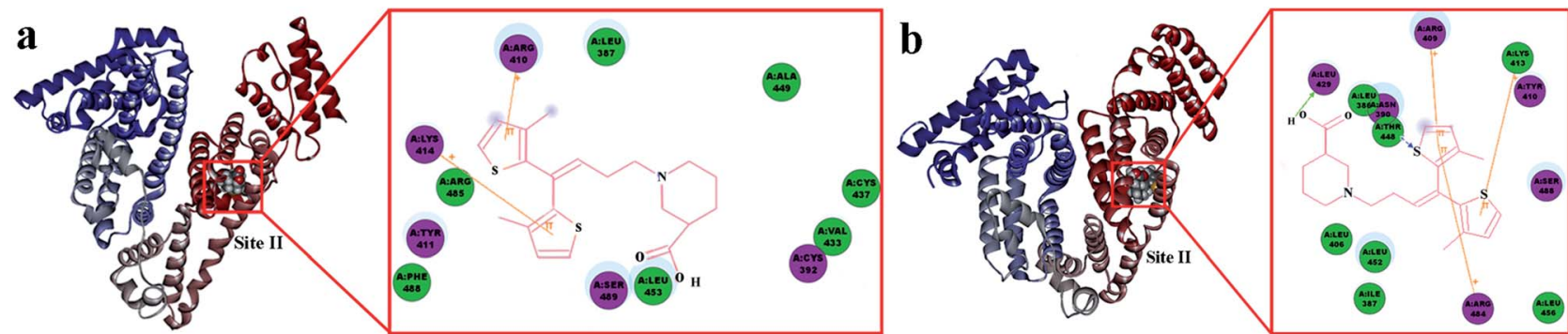

Fig. 6 Surface around TGB in Sudlow site II of HSA (a) and BSA (b), respectively. The inset is the 2D docking modes between TGB and HSA or BSA. Only important interacting residues are shown and represented as sticks. Residues involved in hydrogen bonding, charge, and polar interactions are represented by magenta circles. Residues involved in van der Waals interactions are represented by green circles. The solvent-accessible surfaces of atoms are represented by a blue halo around the atom. The diameter of the circle is proportional to the solvent accessible surface. Cation- $\pi$ interactions are represented by an orange line, whereby connecting positive ions of amino acid residues of HSA or BSA with the thiophene rings of TGB.

of TGB can reduce the fluorescence intensity of dansylsarcosine-BSA but it has no obvious influence on the emission intensity of dansylamide-BSA, indicating that TGB could compete with dansylsarcosine in BSA. However, there is no obvious competition between TGB and dansylamide. Therefore, TGB and dansylsarcosine could bind to the same site (Sudlow site II) of BSA, and addition of TGB could partially replace the dansylsarcosine that bound to BSA.

Subsequently, AutoDock software was used to present the optimal binding model for further binding-orientation analyses of the TGB-HSA complex and TGB-BSA complex (Fig. 6).

In Fig. 6a, the ligand TGB is located inside the protein structure of HSA, particularly, in the hydrophobic cavity in Sudlow site II. It consists of the following amino acids: Lys-414, Arg-485, Tyr411, Phe-488, Arg-410, Leu-387, Ser-489, Leu-453, Ala-449, Cys437, Val-433, and Cys-392. TGB and HSA undergo changes with the influence of cation- $\pi$ interaction, which is a noncovalent molecular interaction between a positively charged molecule and a $\pi$ system, to minimize the space between them. ${ }^{38}$ Panel 2D displays the cation $-\pi$ interactions between HSA and TGB at pH 7.40, as the Lys-414 and Arg-410 took part in forming cation- $\pi$ binding when the cations on these residues were separately incorporated into the $\pi$ system of the two thiophene rings of TGB. Fig. 6b shows the lowest energy molecular modelling of TGB docking to site II of BSA. The following amino acids are around the binding site: Leu-429, Leu-386, Asn-390, Thr-448, Leu406, Leu-452, Ile-387, Arg-484, Leu-456, Arg-409, Lys-413, Tyr-410, and Ser-488. Especially, there are cation- $\pi$ interactions between the thiophene ring of TGB and the Arg-409, Arg-484, and Lys-413 of BSA, and a hydrogen bonding force also exists.

In combination with site marker competitive experiments and molecular docking simulation results, the TGB could steadily bind to Sudlow site II (subdomain IIIA) of HSA and BSA, and the hydrophobic interactions between the thiophene rings of TGB and the hydrophobic amino acid residues of proteins played a major role in the TGB-protein complexation.

\subsection{Evaluation of the conformational changes of HSA and BSA induced by TGB}

3.4.1 3D fluorescence spectroscopy. 3D fluorescence spectroscopy can investigate microenvironmental changes in proteins when combined with small molecules. ${ }^{39}$ Contour maps for, respectively, the TGB-HSA and TGB-BSA complexes with molar ratios of $0: 1$ and $6: 1$ (drug to protein) are shown in Fig. $7 \mathrm{a}-\mathrm{d}$, and their parameters are illustrated in Table 2. Peak 1 $\left(\lambda_{\mathrm{ex}}=225 \mathrm{~nm}, \lambda_{\mathrm{em}}=335 \mathrm{~nm}\right.$ for HSA and $\lambda_{\mathrm{ex}}=225 \mathrm{~nm}, \lambda_{\mathrm{em}}=$ $342 \mathrm{~nm}$ for BSA) mainly exhibits fluorescence characteristics of polypeptide backbone structures. Peak $2\left(\lambda_{\mathrm{ex}}=280 \mathrm{~nm}, \lambda_{\mathrm{em}}=\right.$ $338 \mathrm{~nm}$ for HSA and $\lambda_{\mathrm{ex}}=280 \mathrm{~nm}, \lambda_{\mathrm{em}}=340 \mathrm{~nm}$ for BSA) mainly reveals the spectral behavior of tryptophan and tyrosine residues and reflects the polarity of the HSA microenvironment. ${ }^{40,41}$ By comparing and analyzing the position of the excitation and emission wavelength, and the Stokes shift (Table 2) of TGB-HSA complex in the absence and presence of TGB, it could be seen that both peak 1 and 2 were nearly unchanged, which indicated that the molecular microenvironment around the fluorophore residues of HSA was almost unaffected by TGB. Similarly, the microenvironment of BSA was not obviously perturbed by TGB either, as the Stokes shift of BSA had no significant change.
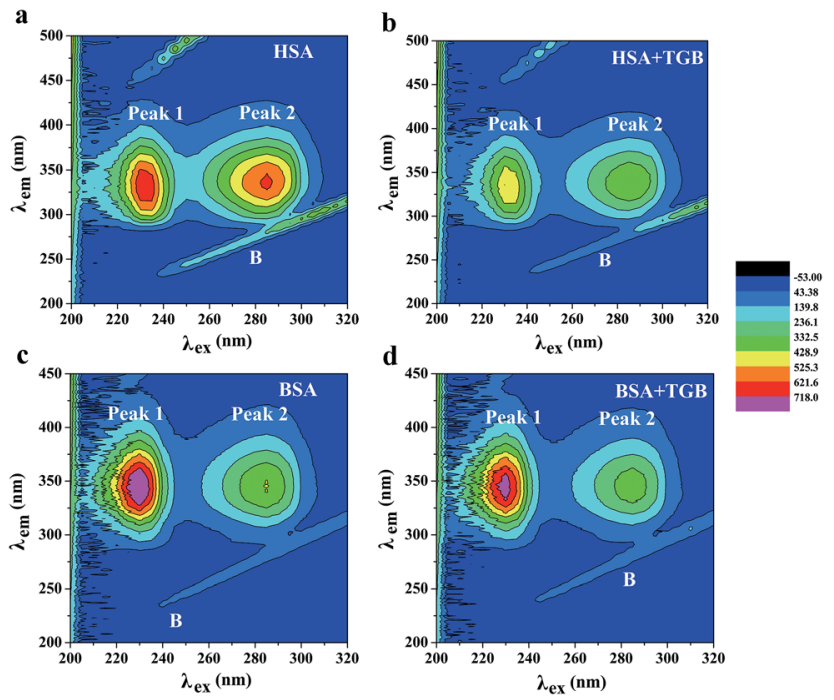

Fig. 7 At pH 7.40 and $298 \mathrm{~K}$, the contour spectra of HSA ( $a$ and $b$ ) and BSA ( $c$ and $d$ ) in the absence and presence of TGB. The concentrations of HSA and BSA are $2.4 \mu \mathrm{M}$ and $0.7 \mu \mathrm{M}$, respectively, and the molar ratios of TGB to HSA or BSA are $0: 1$ and $6: 1$. 
Table 2 3D fluorescence spectral parameters of TGB-HSA and TGB-BSA systems

\begin{tabular}{lllll}
\hline System & Peaks & Peak position $\left[\lambda_{\text {ex }} / \lambda_{\text {em }}\left(\mathrm{nm} \mathrm{nm}^{-1}\right)\right]$ & Stokes shift $[\Delta \lambda(\mathrm{nm})]$ & \multicolumn{1}{c}{ Intensity } \\
\hline HSA & B & $235 / 235 \rightarrow 320 / 320$ & 0 & $71.289 \rightarrow 364.174$ \\
& 1 & $225 / 335$ & 110 & 649.917 \\
TGB-HSA & 2 & $280 / 338$ & 58 & $72.360 \rightarrow 412.557$ \\
& $\mathrm{~B}$ & $225 / 240 \rightarrow 320 / 320$ & 0 & 526.065 \\
BSA & 1 & $280 / 339$ & 111 & 420.584 \\
& 2 & $240 / 240 \rightarrow 320 / 320$ & 59 & $56.465 \rightarrow 124.164$ \\
& $\mathrm{~B}$ & $225 / 342$ & 0 & 872.205 \\
TGB-BSA & 1 & $280 / 340$ & 60 & 434.241 \\
& 2 & $245 / 245 \rightarrow-320 / 320$ & 0 & $58.981 \rightarrow 136.431$ \\
& $\mathrm{~B}$ & $225 / 343$ & 118 & 790.332 \\
& 1 & $280 / 341$ & 61 & 385.896
\end{tabular}

3.4.2 CD spectroscopy. CD spectroscopy is a wellestablished technique for monitoring the influence of ligands on the secondary structure of proteins. ${ }^{42,43}$ Typical CD spectra of HSA and BSA with or without TGB are shown in Fig. 8. Serum proteins HSA and BSA have similar peak shapes, which display
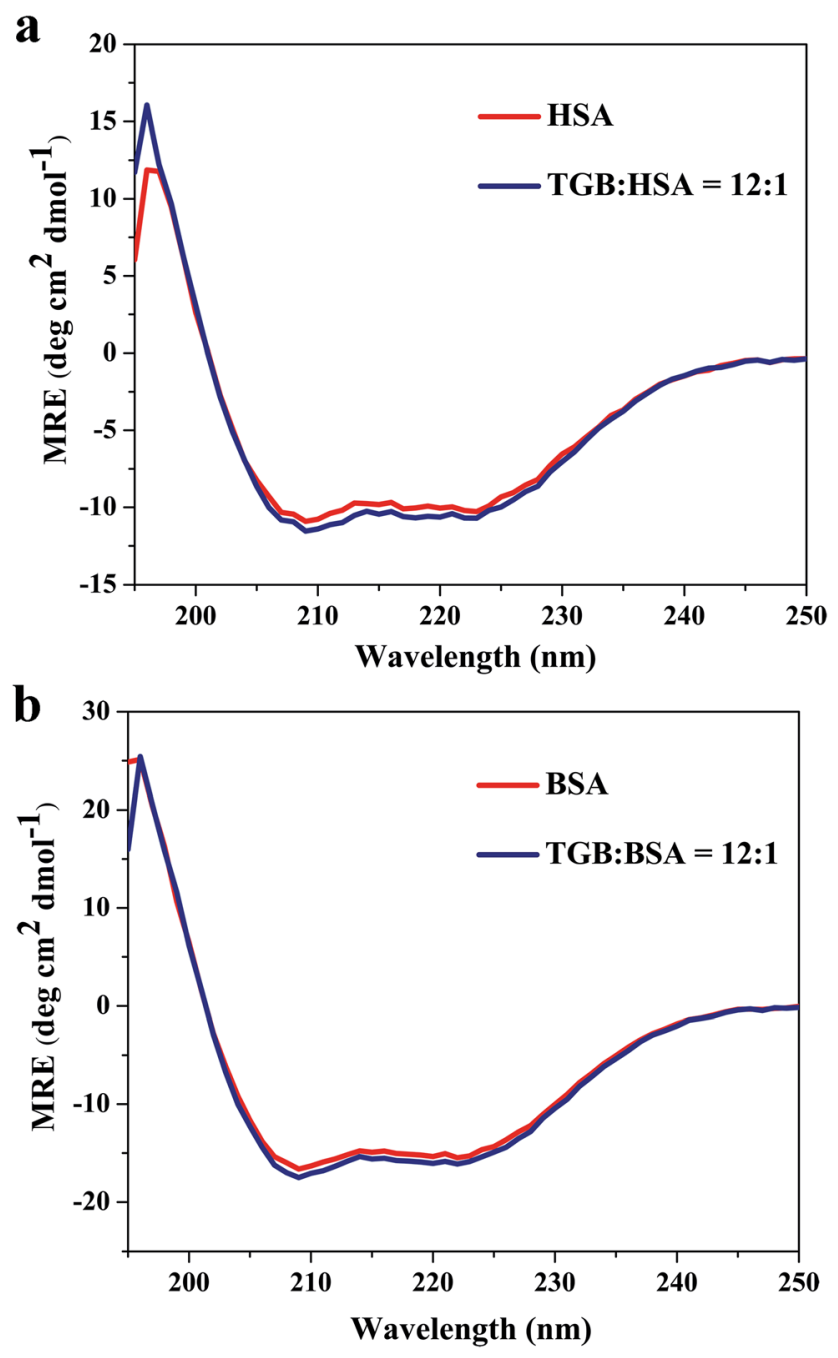

Fig. $8 \mathrm{CD}$ spectra of HSA (a) and BSA (b) with or without TGB at pH 7.40 and $298 \mathrm{~K}$, respectively. The concentrations of HSA and BSA are both $2 \mu \mathrm{M}$, and the molar ratios of TGB to HSA or BSA are $0: 1$ and $12: 1$. two negative bands at 208 and $220 \mathrm{~nm}$ corresponding to $\pi \rightarrow \pi^{*}$ and $n \rightarrow \pi^{*}$ transitions, representing the $\alpha$-helix structure of these proteins. After the addition of TGB, the characteristic peptide CD bonds of HSA and BSA both remained essentially unchanged. This result indicated that TGB did not induce any obvious perturbations of the proteins fold at $298 \mathrm{K.}{ }^{44}$ Therefore, the secondary structure of HSA and BSA were almost unchanged even when induced by TGB. In summary, the TGB had no significant effect on the microenvironment and secondary structure of HSA and BSA.

\section{Conclusion}

In all, this study presented a systematic investigation of the interaction mechanism between TGB and serum albumins, including HSA and BSA, using different calorimetric and spectroscopic techniques. There were no obvious differences between TGB-HSA and TGB-BSA systems. TGB could steadily combine with the proteins and the thermal stabilities of the two serum albumins were slightly enhanced by complexation with TGB. The binding abilities of both the TGB-HSA complex and TGB-BSA complex were moderate. TGB and dansylsarcosine competitively interact with Sudlow site II of HSA and BSA. Hydrophobic interactions were dominant in the formation of TGB-HSA and TGBBSA complexes. The results of molecular docking intuitively showed that the TGB molecule entered the hydrophobic cavity in Sudlow site II of HSA and BSA and formed specific cation- $\pi$ bonds with the surrounding amino acids. Additionally, the conformations of HSA and BSA were almost unaffected by TGB. This study has provided insights into the interaction mechanism between TGB and serum albumins, which could provide useful information for better utilization of TGB.

\section{Conflicts of interest}

There are no conflicts of interest to declare.

\section{Acknowledgements}

This study was supported by the Large-scientific Instruments Sharing Platform Ability Construction of Sichuan Province (Grant No. 2016KJTS0037). Thanks to the State Key Laboratory 
of Biotherapy and the center of Growth, Metabolism and Aging, Sichuan University for the VP-Capillary differential scanning calorimetric (DSC) and isothermal titration calorimeter (ITC) technical assistance. Thanks for the help of Engineering Experimental Teaching Center, School of Chemical Engineering, Sichuan University.

\section{Notes and references}

1 W. Froestl, Future Med. Chem., 2011, 3, 163-175.

2 K. Salat, A. Podkowa, N. Malikowska, F. Kern, J. Pabel, E. Wojcieszak, K. Kulig, K. T. Wanner, B. Strach and E. Wyska, Neuropharmacology, 2017, 113, 331-342.

3 K. Salat, A. Podkowa, P. Kowalczyk, K. Kulig, A. Dziubina, B. Filipek and T. Librowski, Pharmacol. Rep., 2015, 67, 465472.

4 J. Liu, D. Huang, J. Xu, J. Tong, Z. Wang, L. Huang, Y. Yang, X. Bai, P. Wang, H. Suo, Y. Ma, M. Yu, J. Fei and F. Huang, Sci. Rep., 2015, 5, 15720.

5 P. A. Rutecki and B. E. Gidal, Epilepsy Behav., 2002, 3, 24.

6 H. A. Spiller, D. Wiles, J. L. Russell and M. J. Casavant, Hum. Exp. Toxicol., 2016, 35, 109-113.

7 S. Fatima, P. Sen, P. Sneha and C. G. Priyadoss, Appl. Biochem. Biotechnol., 2017, 182, 82-96.

8 A. Bujacz, J. A. Talaj, K. Zielinski, A. J. Pietrzyk-Brzezinska and P. Neumann, Acta Crystallogr., Sect. D, 2017, 73, 896-909.

9 J. H. Shi, D. Q. Pan, X. X. Wang, T. T. Liu, M. Jiang and Q. Wang, J. Photochem. Photobiol., B, 2016, 162, 14-23.

10 K. Gałęcki, K. Hunter, G. Daňková, E. Rivera, L. W. Tung and K. Mc Sherry, J. Lumin., 2016, 177, 235-241.

11 G. A. McMillin and M. D. Krasowski, Therapeutic Drug Monitoring of Newer Antiepileptic Drugs, Clinical Challenges in Therapeutic Drug Monitoring, Elsevier Inc., 2016, pp. 101-134.

12 G. Rabbani, M. H. Baig, E. J. Lee, W. K. Cho, J. Y. Ma and I. Choi, Mol. Pharm., 2017, 14, 1656-1665.

13 E. L. Gelamo, C. H. T. P. Silva, H. Imasato and M. Tabak, Biochim. Biophys. Acta, Protein Struct. Mol. Enzymol., 2002, 1594, 84-99.

14 K. Mikael, S. Robert, E. Svante and A. Bo, Analyst, 1994, 119, 417-419.

15 K. Ray, R. Badugu and J. R. Lakowicz, $R S C A d v .$, 2015, 5, 54403-54411.

16 J. Ghuman, P. A. Zunszain, I. Petitpas, A. A. Bhattacharya, M. Otagiri and S. Curry, J. Mol. Biol., 2005, 353, 38-52.

17 A. Bujacz, K. Zielinski and B. Sekula, Proteins, 2014, 82, 2199-2208.

18 C. J. Layton and H. W. Hellinga, Protein Sci., 2011, 20, 14391450.

19 S. Yasmeen and Riyazuddeen, J. Chem. Thermodyn., 2017, 110, 79-86.

20 S. A. Kantonen, N. M. Henriksen and M. K. Gilson, Biochim. Biophys. Acta, Gen. Subj., 2017, 1861, 485-498.
21 M. Braia, D. Loureiro, G. Tubio, M. E. Lienqueo and D. Romanini, Colloids Surf., B, 2017, 155, 507-511.

22 A. Khammari, A. A. Saboury, M. H. Karimi-Jafari, M. Khoobi, A. Ghasemi, S. Yousefinejad and O. K. Abou-Zied, J. Phys. Chem. C, 2017, 19, 10099-10115.

23 G. Rabbani, M. H. Baig, E. J. Lee, W. K. Cho, J. Y. Ma and I. Choi, Mol. Pharm., 2017, 14, 1656-1665.

24 E. Kaspchak, L. I. Mafra and M. R. Mafra, Food Chem., 2018, 252, 1-8.

25 O. Callies and A. Hernandez Daranas, Nat. Prod. Rep., 2016, 33, 881-904.

26 X. Li and S. Wang, New J. Chem., 2015, 39, 386-395.

27 H. Yang, P. Tang, B. Tang, Y. Huang, X. Xiong and H. Li, RSC Adv., 2017, 7, 10242-10251.

28 Y. Chen, Y. Zhou, M. Chen, B. Xie, J. Yang, J. Chen and Z. Sun, Food Chem., 2018, 258, 393-399.

29 J. C. N. Santos, I. M. da Silva, T. C. Braga, A. de Fatima, I. M. Figueiredo and J. C. C. Santos, Int. J. Biol. Macromol., 2018, 113, 1032-1040.

30 J. R. Lakowics, J. Biomed. Opt., 2008, 13, 277-289.

31 M. Chakraborty, S. Paul, I. Mitra, M. Bardhan, M. Bose, A. Saha and T. Ganguly, J. Photochem. Photobiol., B, 2018, 178, 355-366.

32 V. Dahiya, B. Chaubey, A. K. Dhaharwal and S. Pal, Pestic. Biochem. Physiol., 2017, 139, 92-100.

33 E. Morgunova, S. Saller, I. Haase, M. Cushman, A. Bacher, M. Fischer and R. Ladenstein, J. Biol. Chem., 2007, 282, 17231-17241.

34 P. D. Ross and S. Subramanian, Biochemistry, 2002, 20, 30963102.

35 F. Bou-Abdallah, S. E. Sprague, B. M. Smith and T. R. Giffune, J. Chem. Thermodyn., 2016, 103, 299-309.

36 G. Sudlow, D. J. Birkett and D. N. Wane, Mol. Pharmacol., 1975, 11, 824-832.

37 G. Sudlow, D. J. Birkett and D. N. Wade, Mol. Pharmacol., 1976, 12, 1052-1061.

38 G. U. Nienhaus, Protein-ligand interactions: methods and applications, Humana Press, 2005, pp. 451-492.

39 Z. Chen, H. Xu, Y. Zhu, J. Liu, K. Wang, P. Wang, S. Shang, X. Yi, Z. Wang, W. Shao and S. Zhang, RSC Adv., 2014, 4, 25410-25419.

40 M. S. Ali, M. Amina, H. A. Al-Lohedan and N. M. Al Musayeib, J. Photochem. Photobiol., B, 2018, 182, 9-17.

41 M. S. Ali and H. A. Al-Lohedan, Colloids Surf., B, 2015, 134, 392-400.

42 S. Ikhlas, A. Usman and M. Ahmad, J. Biomol. Struct. Dyn., 2018, 1-11.

43 J. Kaur, L. Katopo, A. Hung, J. Ashton and S. Kasapis, Food Chem., 2018, 252, 163-170.

44 A. Maji, M. Beg, A. K. Mandal, S. Das, P. K. Jha, A. Kumar, S. Sarwar, M. Hossain and P. Chakrabarti, J. Mol. Struct., 2017, 1141, 584-592. 